\title{
Epidemiology and antifungal susceptibility of bloodstream Candida isolates in Quebec: Report on 453 cases between 2003 and 2005
}

\author{
Guy St-Germain $\mathrm{BSC}^{1}$, Michel Laverdière $\mathrm{MD}^{2}$, René Pelletier $\mathrm{MD}^{3}$, Pierre René $\mathrm{MD}^{4}$, \\ Anne-Marie Bourgault $\mathrm{MD}^{5}$, Claude Lemieux $\mathrm{MD}^{5}$, Michael Libman $\mathrm{MD}^{4}$
}

\begin{abstract}
G St-Germain, M Laverdière, R Pelletier, et al. Epidemiology and antifungal susceptibility of bloodstream Candida isolates in Quebec: Report on 453 cases between 2003 and 2005. Can J Infect Dis Med Microbiol 2008;19(1):55-62.
\end{abstract}

BACKGROUND: Between May 2003 and April 2005, a populationbased surveillance of Candida bloodstream infections was conducted in Quebec. A total of 453 episodes of candidemia (464 yeast isolates) from 54 participating hospitals were studied.

RESULTS: The annual incidence rate was three per 100,000 population. Global hospital mortality was $38 \%$. The most common predisposing factors were the presence of an intravascular catheter $(80 \%)$, use of antibacterial therapy (67\%), stay in an intensive care unit (49\%), use of parenteral nutrition (32\%) and intra-abdominal surgery (31\%). Fluconazole alone or in association with other antifungals was used for treatment in over $80 \%$ of cases. Candida albicans comprised $62 \%$ of isolates, followed by Candida glabrata (17\%), Candida parapsilosis (9\%), Candida tropicalis (5\%), Candida lusitaniae (3\%) and Candida krusei (3\%). Of the $288 \mathrm{C}$ albicans isolates, seven (2\%) were resistant to flucytosine, one to fluconazole and none to itraconazole or voriconazole. Of the 75 non-C albicans species isolates with reduced susceptibility to fluconazole (minimum inhibitory concentration [MIC] $16 \mu \mathrm{g} / \mathrm{mL}$ or greater), none were susceptible to itraconazole (MIC $0.12 \mathrm{mg} / \mathrm{L}$ or lower), whereas 71 (95\%) were susceptible to voriconazole (MIC $1 \mu \mathrm{g} / \mathrm{mL}$ or lower). However, only five of 12 (42\%) fluconazole-resistant isolates were susceptible to voriconazole. Posaconazole, ravuconazole and caspofungin displayed a broad spectrum of activity against these isolates, with MICs of $1 \mathrm{mg} / \mathrm{L}$ or lower in $56 \%$, $92 \%$ and $100 \%$ of isolates, respectively. Overall, a correlation $\left(\mathrm{r}^{2}>0.87\right)$ was observed among increasing fluconazole MICs and the geometric mean MICs of itraconazole, voriconazole, posaconazole and ravuconazole. CONCLUSIONS: These surveillance results when compared with those of the 1993 to 1995 survey confirm little variation in the distribution of species causing invasive Candida infection over a 10-year period in Quebec, as well as the continuous excellent overall in vitro activity of fluconazole.

Key Words: Antifungal resistance; Candidemia; Surveillance

$\mathrm{T}$ he increasing incidence of nosocomial fungal infections observed over the past decades is mainly caused by the growing population of patients undergoing treatment for severe underlying medical conditions associated with concomitant use of antibiotics and intensive care support $(1,2)$. A Candida bloodstream infection carries a significant risk of death, and strongly impacts on the length of hospital stay and

\section{Épidémiologie des candidémies et leur sensibilité aux antifongiques : Rapport de 453 cas recensés entre 2003 et 2005 au Québec}

HISTORIQUE : Entre mai 2003 et avril 2005, une surveillance des cas de candidémie dans la population a été réalisée au Québec. En tout, 453 épisodes de candidémie (464 isolats de levures) provenant de 54 hôpitaux participants ont été analysés.

RÉSULTATS : L'incidence annuelle a été évaluée à trois (3) cas par 100000 de population. La mortalité hospitalière globale a été de $38 \%$. Les principaux facteurs prédisposants étaient la présence d'un cathéter intravasculaire ( $80 \%)$, l'utilisation d'antibactériens (67\%), un séjour en unité de soins intensifs (49\%), la nutrition parentérale (32\%) et la chirurgie abdominale $(31 \%)$. Le fluconazole, en monothérapie ou associé à d'autres antifongiques, a été utilisé pour le traitement de plus de $80 \%$ des cas. Candida albicans représentait $62 \%$ des isolats, suivi de Candida glabrata (17\%), Candida parapsilosis (9 \%), Candida tropicalis (5\%), Candida lusitaniæ (3 \%) et Candida krusei (3 \%). Parmi les 288 isolats de C. albicans, sept $(2 \%)$ se sont révélés résistants à la flucytosine, un au fluconazole et aucun n'a été résistant à l'itraconazole ou au voriconazole. Parmi les 75 isolats non albicans moins sensibles au fluconazole (concentrations minimales inhibitrices [CMI] $16 \mu \mathrm{g} / \mathrm{mL}$ ou plus), aucun n'a été sensible à l'itraconazole (CMI 0,12 mg/L ou moins), tandis que 71 (95\%) ont été sensibles au voriconazole (CMI $1 \mu \mathrm{g} / \mathrm{mL}$ ou moins). Par contre, seulement cinq des isolats résistants au fluconazole sur 12 (42\%) se sont révélés sensibles au voriconazole. Le posaconazole, le ravuconazole et la capsofungine ont manifesté un large spectre d'activité contre ces isolats, avec des CMI de $1 \mathrm{mg} / \mathrm{L}$ ou moins chez $56 \%, 92 \%$ et $100 \%$ des isolats, respectivement. Dans l'ensemble, on a pu observer une corrélation $\left(r^{2}>0,87\right)$ entre les CMI croissantes du fluconazole et les moyennes géométriques des CMI de l'itraconazole, du voriconazole, du posaconazole et du ravuconazole.

CONCLUSIONS : Comparativement aux résultats de la surveillance exercée entre 1993 à 1995, cette analyse confirme que la distribution des espèces de Candida responsables d'infections invasives a peu varié au Québec en dix ans et que le fluconazole conserve une excellente activité globale in vitro.

${ }^{1}$ Laboratoire de santé publique/Institut national de santé publique du Québec; ${ }^{2} \mathrm{H}$ ôpital Maisonneuve-Rosemont; ${ }^{3}$ Centre hospitalier universitaire de Québec; ${ }^{4} \mathrm{McGill}$ University Health Center; ${ }^{5}$ Centre hospitalier de l'Université de Montréal, Québec City, Québec

Correspondence: Mr Guy St-Germain, Laboratoire de santé publique du Québec, 20045 chemin Sainte-Marie, Sainte-Anne-de-Bellevue,

Québec H9X 3R5. Telephone 514-457-2070, fax 514-457-6346, e-mail guy.st-germain@inspq.qc.ca

Received for publication June 11, 2007. Accepted August 9, 2007 
the fact that most studies have been performed in single centres (14-17). The present study follows in the footsteps of two previous studies $(18,19)$ performed in 1985 and 1996 in the province of Quebec. The main objectives were to provide insight into the incidence, species distribution and resistance profiles of Candida species causing bloodstream infections in a large unselected population, and to detect variations over the years.

\section{PATIENTS AND METHODS}

\section{Surveillance}

The data were collected over the course of a two-year surveillance program between May 2003 and April 2005. Strains (one strain per species per patient) of Candida isolated from blood in hospital laboratories throughout Quebec were sent to the provincial reference laboratory. An episode was defined as a single positive blood culture with any Candida species; candidemias occurring in the same patient more than 30 days after an initial positive episode were considered to be a new episode. Demographic and clinical data were recorded on a standardized case report form and included age, sex, site of isolation, infectious diagnosis, underlying conditions, predisposing factors, history of exposure to antifungal agents pre- and postdetection of the isolates, central venous catheter withdrawal and culture, and clinical outcome.

\section{Organism identification}

Candida albicans and Candida dubliniensis isolates were recognized by germ tube analysis and growth characteristics on CHROMagar Candida medium (Dalynn Biologicals Inc, Canada). Differentiation of $\mathrm{C}$ dubliniensis from $\mathrm{C}$ albicans was initially performed on Staib agar, and was later confirmed by biochemical tests and sequencing of the D1/D2 region of large subunit 28S ribosomal RNA (20). Non-C albicans-dubliniensis isolates were identified by morphology evaluation on cornmeal Tween 80 agar, carbohydrate assimilation tests using API ID $32 \mathrm{C}$ strips (bioMérieux Inc, USA), urease activity, sugar fermentation and supplemental tests when needed.

\section{Drugs}

Amphotericin B (Bristol-Myers Squibb, USA), flucytosine (Sigma-Aldrich Inc, USA), fluconazole (Pfizer Canada), itraconazole (Janssen Pharmaceutica, USA), voriconazole (Pfizer Canada), ravuconazole (Bristol-Myers Squibb, USA), posaconazole (Schering-Plough, USA) and caspofungin (Merck-Frosst Canada) were obtained from their respective manufacturers as standard powders. Diluted concentrations were distributed in flat-bottomed, 96-well microdilution plates and stored in plastic bags at $-70^{\circ} \mathrm{C}$ until needed.

\section{Susceptibility testing}

Testing was performed at the Laboratoire de santé publique du Québec (Quebec City, Quebec), by a broth microdilution method as described in procedure M27-A2 of the Clinical and Laboratory Standards Institute (CLSI), with minor modifications (21). In each well, $50 \mu \mathrm{L}$ of inoculum were added to $50 \mu \mathrm{L}$ of broth containing the antifungals, for a total volume of $100 \mu \mathrm{L}$ (CLSI method recommends $200 \mu \mathrm{L}$ ). The smaller volume was used to avoid any spillage or well-to-well contamination, while mechanically agitating the plates before reading. The culture media used were RPMI 1640 for flucytosine, the azoles and caspofungin, and M3 broth supplemented with 2\% glucose for amphotericin B $(21,22)$. Inhibitory concentrations were determined visually and spectrophotometrically, after both $24 \mathrm{~h}$ and $48 \mathrm{~h}$ of incubation in ambient air at $35^{\circ} \mathrm{C}(18)$. Before reading at both $24 \mathrm{~h}$ and $48 \mathrm{~h}$, the plates were agitated for 2 min at $900 \mathrm{rpm}$ with a shaker (SLT Lab Instruments, model EAS 2/4, Grödig, Austria). They were then examined with a reading mirror and the growth in each well was compared with the growth control well. Amphotericin B minimum inhibitory concentrations (MICs) were determined as $100 \%$ inhibition. For all other drugs, the MICs corresponded to the concentration at which a prominent decrease in growth relative to the growth control well was observed. Furthermore, the optical density of the growth in each well was determined with the use of an automatic plate reader set at $495 \mathrm{~nm}$ (Pasteur Diagnostic LP400, Adil Instruments, France). The MIC for amphotericin B was determined as the lowest drug concentration with an optical density corresponding to lower than 0.01 in turbidity or to a $50 \%$ decrease in turbidity compared with that of the growth control. In the present report, visually read MICs were given precedence over spectrophotometric results. Spectrophotometrically measured MICs were used to establish a cut-off point when trailing growth occurred. The MICs reported in the present study are those read at $48 \mathrm{~h}$ of incubation for all antifungals, with the exceptions of amphotericin B and caspofungin which were read at $24 \mathrm{~h}$ (22-24). Quality control was performed by testing Candida parapsilosis ATCC 22019 and Candida krusei ATCC 6258 with each set of clinical isolates (21). Candida lusitaniae, ATCC 200950 (5W31), was also tested repeatedly as an indicator of amphotericin B resistance $(22,25)$.

\section{Analysis of results}

The rate of incidence was based on a total population of 7.5 million, as determined for 2004 by Statistics Canada estimates. Interpretive breakpoints were applied only to flucytosine, itraconazole, fluconazole and voriconazole following CLSI recommendations $(21,26)$. Guidelines for amphotericin $\mathrm{B}$, ravuconazole, posaconazole and caspofungin have not yet been established. Relations between proportions were analyzed by $\chi^{2}$ tests using Epi Calc 1.02 (Centers for Disease Control and Prevention and World Health Organization). A two-sided $\mathrm{P}$ value of less than 0.05 was used to determine statistical significance. The correlation coefficient, $\mathrm{r}^{2}$, used to measure the relationship among MICs of fluconazole and geometric mean MICs of voriconazole, itraconazole, posaconazole and ravuconazole, was calculated with Excel (Microsoft Corporation, USA) functions, using the $\log _{10}$ of the MICs.

\section{RESULTS}

\section{Patient population and clinical data}

A total of 453 episodes of candidemia were reported through the participation of 54 hospital laboratories. These laboratories included all the institutions offering services in medical mycology in Quebec. Thirteen of these were university-affiliated and contributed $55 \%$ of all specimens. Overall, the number of isolates ranged from one to 56 per institution, with a median number of four isolates. The average annual incidence of candidemia for the province of Quebec was three cases per 100,000 population. Bloodstream infections were diagnosed in 240 male and 213 female patients, with ages ranging from one day to 97 years (mean of 57.5 years, median of 62.4 years). Clinical data questionnaires were available for 361 patients (Table 1). The crude mortality rate was $37.6 \%$ (115 of 306). Major predisposing factors were central venous catheter $79.9 \%$ 
(266 of 333), antimicrobial therapy 67.3\% (224 of 333) and stay in intensive care unit 49.2\% (164 of 333). Overall, candidemias were treated with fluconazole alone or in combination in $80.1 \%$ of cases, while amphotericin B and caspofungin were used in $23.2 \%$ and $19.5 \%$ of patients, respectively. Among the 272 patients known to have received antifungal therapy, fluconazole monotherapy was used in $62.1 \%$ of cases. Fluconazole had been administered to $7.8 \%$ of patients within the 30 days preceding the onset of candidemia. Forty-seven patients were reported as not having been treated with any antifungal agent; the mortality rate in this group was $60.5 \%$.

\section{Etiology}

A total of 464 Candida isolates were received for analysis and the overall distribution of species is shown in Table 2. The four most frequently isolated species were C albicans (62\%), Candida glabrata (17\%), C parapsilosis (9\%) and Candida tropicalis $(4.5 \%)$. Two different Candida species were isolated from the same patient in 11 of the 453 cases. Two patients had a recurrent episode. The frequency of isolation of $\mathrm{C}$ parapsilosis decreased with age, from $19.4 \%$ in infants younger than one year of age to $8.0 \%$ in patients 65 years of age or older. However, this difference did not reach statistical significance $(\mathrm{P}>0.05)$. In contrast, $\mathrm{C}$ glabrata was seen less often in neonates $(3.2 \%)$ than in the elderly $(21.6 \%) \quad(\mathrm{P}<0.05)$. Notably, nine of 13 isolates of C lusitaniae (69.2\%) were from these two age groups. Among the Candida isolates recovered from the 25 patients treated with fluconazole before developing candidemia, nine (36\%) were C albicans, six (24.0\%) C glabrata, six $(24.0 \%) \mathrm{C}$ krusei, three $(12.0 \%) \mathrm{C}$ parapsilosis and one $(4.0 \%) \mathrm{C}$ tropicalis. Three $(0.6 \%)$ isolates were identified as $\mathrm{C}$ dubliniensis in the course of the present study.

\section{Susceptibility to antifungals}

All 464 isolates were tested for susceptibility to amphotericin B, flucytosine, fluconazole, itraconazole, voriconazole, ravuconazole, posaconazole and caspofungin (Table 2). Given the testing conditions used in the present study, resistance to amphotericin B appeared to be rare. MICs $2 \mathrm{mg} / \mathrm{L}$ or greater determined after $24 \mathrm{~h}$ of incubation were observed in one isolate each of $\mathrm{C}$ albicans, $\mathrm{C}$ krusei and C lusitaniae. Only one of 13 isolates of C lusitaniae, a recognized amphotericin B-resistant species, had a MIC of $2 \mathrm{mg} / \mathrm{L}$ or greater. Resistance to flucytosine was mainly observed in isolates of $\mathrm{C}$ krusei (8.3\%) and C tropicalis (9.5\%).

A very large proportion (98.9\%) of C albicans, C parapsilosis and $\mathrm{C}$ tropicalis isolates were susceptible to fluconazole. All $\mathrm{C}$ krusei strains were considered resistant to this drug irrespective of MIC results (21). Seven (1.6\%) isolates of Candida species, other than $\mathrm{C}$ krusei, were resistant to fluconazole (MICs $64 \mathrm{mg} / \mathrm{L}$ or greater). These included four isolates of $\mathrm{C}$ glabrata and one each of $\mathrm{C}$ albicans, $\mathrm{C}$ tropicalis and Candida pelliculosa. The C albicans strain originated from an AIDS patient previously treated with fluconazole, before the onset of candidemia. The $\mathrm{C}$ tropicalis strain was isolated from a patient during a second episode of candidemia, the first of which had been treated with fluconazole two months earlier. Another 58 Candida species isolates (one isolate each of $\mathrm{C}$ albicans, $\mathrm{C}$ parapsilosis and Candida guilliermondii and $55 \mathrm{C}$ glabrata), other than C krusei exhibited fluconazole MICs of $16 \mathrm{mg} / \mathrm{L}$ to $32 \mathrm{mg} / \mathrm{L}$. Both isolates of $\mathrm{C}$ albicans and $\mathrm{C}$ parapsilosis were from patients exposed to fluconazole before the diagnosis of candidemia. Excluding two isolates of $\mathrm{C}$ tropicalis exhibiting a 'low-high'
TABLE 1

Demographic and clinical characteristics of candidemia cases

\begin{tabular}{|c|c|}
\hline Characteristic & $\mathrm{n}(\%)$ \\
\hline \multicolumn{2}{|l|}{ Age, years $(n=453)$} \\
\hline$<1$ & $31(6.8)$ \\
\hline $1-18$ & $9(2.0)$ \\
\hline $19-49$ & $80(17.7)$ \\
\hline $50-64$ & $120(26.5)$ \\
\hline$\geq 65$ & $213(47.0)$ \\
\hline Fever/chills/hypotension ( $n=248)$ & $224(90.3)$ \\
\hline \multicolumn{2}{|l|}{ Underlying disease and conditions ( $n=282$ ) } \\
\hline Diabetes mellitus & $59(20.9)$ \\
\hline Cancer & $52(18.4)$ \\
\hline Hematological malignancy & $27(9.6)$ \\
\hline Neonate & $13(4.6)$ \\
\hline \multicolumn{2}{|l|}{ Organ diseases } \\
\hline Liver & $11(3.9)$ \\
\hline Pulmonary & $11(3.9)$ \\
\hline Cardiac & $15(5.3)$ \\
\hline Renal & $17(6.0)$ \\
\hline Gastrointestinal & $33(11.7)$ \\
\hline Metabolic/autoimmune & $13(4.6)$ \\
\hline HIV infection & $5(1.8)$ \\
\hline Multiple trauma & $3(1.1)$ \\
\hline Intravenous drug user & $4(1.4)$ \\
\hline \multicolumn{2}{|l|}{ Risk factor and intervention $(n=333)$} \\
\hline Central venous catheter & $266(79.9)$ \\
\hline Antimicrobial therapy & $224(67.3)$ \\
\hline Intensive care unit at time of diagnosis & $164(49.2)$ \\
\hline Total parenteral nutrition & $106(31.8)$ \\
\hline Intra-abdominal surgery & $104(31.8)$ \\
\hline Corticosteroid therapy & $61(18.3)$ \\
\hline Ablative chemotherapy & $55(16.5)$ \\
\hline Recent surgery & $45(13.5)$ \\
\hline Dialysis & $6(1.8)$ \\
\hline Hematopoietic stem cell transplantation & $6(1.8)$ \\
\hline Organ transplantation & $4(1.2)$ \\
\hline \multicolumn{2}{|c|}{ Antifungal treatment before onset of candidemia $(n=321)$} \\
\hline Fluconazole & $25(7.8)$ \\
\hline Itraconazole & $1(0.3)$ \\
\hline \multicolumn{2}{|c|}{ Antifungal treatment after onset of candidemia $(n=272)$} \\
\hline Fluconazole & $218(80.1)$ \\
\hline Amphotericin B (lipid formulation included) & $63(23.2)$ \\
\hline Caspofungin & $53(19.5)$ \\
\hline None & $47(17.3)$ \\
\hline \multicolumn{2}{|l|}{ Central venous catheter $(n=333)$} \\
\hline Present & $266(79.9)$ \\
\hline Removed & $221(83.1)$ \\
\hline Not removed & $34(12.8)$ \\
\hline Cultured & $198(89.6)$ \\
\hline Positive & $95(48.0)$ \\
\hline \multicolumn{2}{|l|}{ Evolution } \\
\hline Control blood cultures negative $(n=224)$ & $208(92.9)$ \\
\hline Resolution of signs and symptoms ( $n=257$ ) & $213(82.9)$ \\
\hline Mortality $(n=306)$ & $115(37.6)$ \\
\hline
\end{tabular}


TABLE 2

Antifungal susceptibilities of $\mathbf{4 6 4}$ bloodstream Candida isolates

\begin{tabular}{|c|c|c|c|c|c|c|c|c|c|c|c|c|c|c|c|c|c|c|}
\hline \multirow[b]{2}{*}{ Antifungal agent } & \multicolumn{16}{|c|}{ Number of isolates with minimum inhibitory concentration (MIC; mg/L) of: } & \multirow[b]{2}{*}{ S-DD/I* (\%) } & \multirow[b]{2}{*}{$\mathbf{R}^{\dagger}(\%)$} \\
\hline & 0.008 & 0.016 & 0.03 & 0.06 & 0.12 & 0.25 & 0.5 & 1 & 2 & 4 & 8 & 16 & 32 & 64 & 128 & $>128$ & & \\
\hline \multicolumn{19}{|c|}{ Candida albicans $(\mathrm{n}=288)$} \\
\hline Amphotericin B & - & - & - & 1 & 25 & 122 & 122 & 17 & 1 & - & - & - & - & - & - & - & - & - \\
\hline Flucytosine & - & - & - & 31 & 151 & 56 & 16 & 22 & 2 & 1 & 2 & - & - & 7 & - & - & 0.7 & 2.4 \\
\hline Fluconazole & - & - & - & - & - & 268 & 13 & 1 & 1 & 2 & 1 & - & 1 & 1 & - & - & 0.3 & 0.3 \\
\hline Itraconazole & 2 & 79 & 150 & 42 & 12 & 1 & 2 & - & - & - & - & - & - & - & - & - & 1.0 & 0.0 \\
\hline Voriconazole & 246 & 27 & 11 & 2 & - & 2 & - & - & - & - & - & - & - & - & - & - & 0.0 & 0.0 \\
\hline Ravuconazole & 270 & 10 & - & 5 & - & 2 & - & - & - & - & - & - & - & - & - & - & - & - \\
\hline Posaconazole & 27 & 117 & 97 & 32 & - & 3 & 1 & - & - & - & - & - & - & - & - & - & - & - \\
\hline Caspofungin & - & - & - & 29 & 184 & 72 & 3 & - & - & - & - & - & - & - & - & - & - & - \\
\hline \multicolumn{19}{|c|}{ Candida glabrata $(\mathrm{n}=78)$} \\
\hline Amphotericin B & - & - & - & - & 2 & 7 & 35 & 34 & - & - & - & - & - & - & - & - & - & - \\
\hline Flucytosine & - & - & - & 54 & 23 & - & 1 & - & - & - & - & - & - & - & - & - & 0.0 & 0.0 \\
\hline Fluconazole & - & - & - & - & - & 1 & 1 & 1 & 2 & 5 & 9 & 43 & 12 & 3 & 1 & - & 70.5 & 5.1 \\
\hline Itraconazole & - & - & - & - & 2 & 1 & 10 & 33 & 17 & 9 & 6 & - & - & - & - & - & 14.1 & 83.3 \\
\hline Voriconazole & 1 & - & 2 & 2 & 4 & 10 & 34 & 21 & 3 & 1 & - & - & - & - & - & - & 3.8 & 1.3 \\
\hline Ravuconazole & 1 & 1 & 2 & 2 & 7 & 12 & 21 & 27 & 3 & 2 & - & - & - & - & - & - & - & - \\
\hline Posaconazole & - & - & - & - & 5 & 2 & 9 & 24 & 33 & 2 & - & - & - & - & - & - & - & - \\
\hline Caspofungin & - & - & - & - & 33 & 35 & 10 & - & - & - & - & - & - & - & - & - & - & - \\
\hline \multicolumn{19}{|c|}{ Candida parapsilosis $(n=43)$} \\
\hline Amphotericin B & - & - & 1 & 5 & 15 & 19 & 3 & - & - & - & - & - & - & - & - & - & - & - \\
\hline Flucytosine & - & - & - & 13 & 25 & 2 & 1 & 1 & - & - & - & 1 & - & - & - & - & 2.3 & 0.0 \\
\hline Fluconazole & - & - & - & - & - & 1 & 8 & 14 & 17 & 1 & 1 & - & 1 & - & - & - & 2.3 & 0.0 \\
\hline Itraconazole & - & 2 & 2 & 7 & 19 & 11 & 2 & - & - & - & - & - & - & - & - & - & 30.2 & 0.0 \\
\hline Voriconazole & 3 & 7 & 11 & 17 & 3 & 1 & 1 & - & - & - & - & - & - & - & - & - & 0.0 & 0.0 \\
\hline Ravuconazole & 6 & 12 & 17 & 8 & - & - & - & - & - & - & - & - & - & - & - & - & - & - \\
\hline Posaconazole & 2 & 1 & 3 & 20 & 16 & 1 & - & - & - & - & - & - & - & - & - & - & - & - \\
\hline Caspofungin & - & - & - & - & 2 & 11 & 29 & - & - & - & - & - & - & - & - & - & - & - \\
\hline \multicolumn{19}{|c|}{ Candida tropicalis $(\mathrm{n}=21)$} \\
\hline Amphotericin B & - & - & - & - & 7 & 8 & 5 & 1 & - & - & - & - & - & - & - & - & - & - \\
\hline Flucytosine & - & - & - & 5 & 4 & 3 & 4 & 1 & 2 & - & - & - & 1 & 1 & - & - & 0.0 & 9.5 \\
\hline Fluconazole & - & - & - & - & - & 3 & $3^{\ddagger}$ & $5^{\ddagger}$ & 7 & 2 & - & - & - & 1 & - & - & 0.0 & 4.8 \\
\hline Itraconazole & - & - & 2 & 5 & 8 & $1 \ddagger$ & 2 & 3 & - & - & - & - & - & - & - & - & 14.3 & 14.3 \\
\hline Voriconazole & - & 1 & $5^{\ddagger}$ & 5 & 4 & 3 & 2 & - & - & 1 & - & - & - & - & - & - & 0.0 & 4.8 \\
\hline Ravuconazole & 6 & 4 & $2^{\ddagger}$ & 3 & 1 & - & 2 & 1 & 2 & - & - & - & - & - & - & - & - & - \\
\hline Posaconazole & - & 1 & $4^{\ddagger}$ & 8 & 1 & 3 & 2 & 1 & - & - & - & - & - & - & - & - & - & - \\
\hline Caspofungin & - & - & - & 2 & 8 & 10 & 1 & - & - & - & - & - & - & - & - & - & - & - \\
\hline \multicolumn{19}{|c|}{ Candida lusitaniae $(n=13)$} \\
\hline Amphotericin B & - & - & - & - & - & 2 & 5 & 5 & 1 & - & - & - & - & - & - & - & - & - \\
\hline Flucytosine & - & - & - & 13 & - & - & - & - & - & - & - & - & - & - & - & - & 0.0 & 0.0 \\
\hline Fluconazole & - & - & - & - & - & 1 & 2 & 7 & 3 & - & - & - & - & - & - & - & 0.0 & 0.0 \\
\hline Itraconazole & - & - & - & 2 & 6 & 2 & 3 & - & - & - & - & - & - & - & - & - & 38.5 & 0.0 \\
\hline Voriconazole & 8 & 3 & 1 & 1 & - & - & - & - & - & - & - & - & - & - & - & - & 0.0 & 0.0 \\
\hline Ravuconazole & 2 & 5 & 5 & - & 1 & - & - & - & - & - & - & - & - & - & - & - & - & - \\
\hline Posaconazole & - & - & 4 & 8 & 1 & - & - & - & - & - & - & - & - & - & - & - & - & - \\
\hline Caspofungin & - & - & - & - & - & 4 & 9 & - & - & - & - & - & - & - & - & - & - & - \\
\hline \multicolumn{19}{|c|}{ Candida krusei $(n=12)$} \\
\hline Amphotericin B & - & - & - & - & - & - & 4 & 7 & 1 & - & - & - & - & - & - & - & - & - \\
\hline Flucytosine & - & - & - & - & - & - & - & - & - & - & 4 & 7 & 1 & - & - & - & 91.7 & 8.3 \\
\hline Fluconazole & - & - & - & - & - & - & - & - & - & - & - & 1 & 7 & 3 & 1 & - & - & $100.0 \S$ \\
\hline Itraconazole & - & - & - & - & - & 1 & 8 & 2 & 1 & - & - & - & - & - & - & - & 75.0 & 25.0 \\
\hline Voriconazole & - & - & - & - & - & 4 & 7 & 1 & - & - & - & - & - & - & - & - & 0.0 & 0.0 \\
\hline Ravuconazole & - & - & 1 & 1 & 2 & 5 & 3 & - & - & - & - & - & - & - & - & - & - & - \\
\hline & & & & & & & & & & & & & & & & Contin & ed on ne & page \\
\hline
\end{tabular}


TABLE 2 - CONTINUED

Antifungal susceptibilities of 464 bloodstream Candida isolates

\begin{tabular}{|c|c|c|c|c|c|c|c|c|c|c|c|c|c|c|c|c|c|c|}
\hline \multirow[b]{2}{*}{ Antifungal agent } & \multicolumn{16}{|c|}{ Number of isolates with minimum inhibitory concentration (MIC; mg/L) of: } & \multirow[b]{2}{*}{$\mathrm{S}-\mathrm{DD} / \mathrm{I}^{*}(\%)$} & \multirow[b]{2}{*}{$\mathbf{R}^{\dagger}(\%)$} \\
\hline & 0.008 & 0.016 & 0.03 & 0.06 & 0.12 & 0.25 & 0.5 & 1 & 2 & 4 & 8 & 16 & 32 & 64 & 128 & $>128$ & & \\
\hline \multicolumn{19}{|c|}{ Candida krusei $(\mathrm{n}=12)$ - continued } \\
\hline Posaconazole & - & - & - & - & 2 & 4 & 3 & 3 & - & - & - & - & - & - & - & - & - & - \\
\hline Caspofungin & - & - & - & - & - & 5 & 7 & - & - & - & - & - & - & - & - & - & - & - \\
\hline \multicolumn{19}{|c|}{ Candida dubliniensis $(\mathrm{n}=3)$} \\
\hline Amphotericin B & - & - & - & - & - & 3 & - & - & - & - & - & - & - & - & - & - & - & - \\
\hline Flucytosine & - & - & - & 3 & - & - & - & - & - & - & - & - & - & - & - & - & - & - \\
\hline Fluconazole & - & - & - & - & - & 3 & - & - & - & - & - & - & - & - & - & - & - & - \\
\hline Itraconazole & - & - & 1 & 2 & - & - & - & - & - & - & - & - & - & - & - & - & - & - \\
\hline Voriconazole & 3 & - & - & - & - & - & - & - & - & - & - & - & - & - & - & - & - & - \\
\hline Ravuconazole & 3 & - & - & - & - & - & - & - & - & - & - & - & - & - & - & - & - & - \\
\hline Posaconazole & - & - & 3 & - & - & - & - & - & - & - & - & - & - & - & - & - & - & - \\
\hline Caspofungin & - & - & - & - & 1 & 2 & - & - & - & - & - & - & - & - & - & - & - & - \\
\hline \multicolumn{19}{|c|}{ Other species" $(n=6)$} \\
\hline Amphotericin B & - & - & - & - & 3 & 3 & - & - & - & - & - & - & - & - & - & - & - & - \\
\hline Flucytosine & - & - & - & 4 & 2 & - & - & - & - & - & - & - & - & - & - & - & - & - \\
\hline Fluconazole & - & - & - & - & - & - & 1 & - & 1 & 1 & 1 & 1 & - & 1 & - & - & - & - \\
\hline Itraconazole & - & - & - & - & 1 & 1 & 2 & 2 & - & - & - & - & - & - & - & - & - & - \\
\hline Voriconazole & - & 1 & - & 1 & 2 & 1 & - & 1 & - & - & - & - & - & - & - & - & - & - \\
\hline Ravuconazole & - & 1 & - & 2 & 1 & 1 & 1 & - & - & - & - & - & - & - & - & - & - & - \\
\hline Posaconazole & - & - & - & 1 & 1 & 2 & 1 & 1 & - & - & - & - & - & - & - & - & - & - \\
\hline Caspofungin & - & - & - & - & 1 & 2 & 3 & - & - & - & - & - & - & - & - & - & - & - \\
\hline
\end{tabular}

${ }^{*}$ Susceptible dose-dependant/intermediate (S-DD/l) breakpoints - fluconazole MIC of $16 \mathrm{mg} / \mathrm{L}$ to $32 \mathrm{mg} / \mathrm{L}$, itraconazole $0.25 \mathrm{mg} / \mathrm{L}$ to $0.5 \mathrm{mg} / \mathrm{L}$, flucytosine $8 \mathrm{mg} / \mathrm{L}$ to $16 \mathrm{mg} / \mathrm{L}$, voriconazole $2 \mathrm{mg} / \mathrm{L}$; ${ }^{\dagger}$ Resistance $(R)$ breakpoint - fluconazole MIC of $64 \mathrm{mg} / \mathrm{L}$ or greater, itraconazole $1 \mathrm{mg} / \mathrm{L}$ or greater, flucytosine $32 \mathrm{mg} / \mathrm{L}$ or greater, voriconazole $4 \mathrm{mg} / \mathrm{L}$ or greater; ' ${ }^{`} L \mathrm{Low}$-high' phenomenon observed in one isolate which was categorized as susceptible; $s \mathrm{C}$ krusei is considered inherently resistant to fluconazole regardless of the MIC obtained; T/ncludes Candida guilliermondii (three isolates), Candida pelliculosa (two isolates) and Candida kefyr (one isolate)

phenomenon with azoles, a total of 75 isolates (16.6\%, including C krusei) presented fluconazole MICs of $16 \mathrm{mg} / \mathrm{L}$ or greater; of these isolates, $75(100 \%)$ and five $(6.7 \%)$ exhibited reduced susceptibility to itraconazole (MIC $0.25 \mathrm{mg} / \mathrm{L}$ or greater) and voriconazole (MIC $2 \mathrm{mg} / \mathrm{L}$ or greater), respectively. Ravuconazole and posaconazole MICs of $2 \mathrm{mg} / \mathrm{L}$ or greater were observed for seven (9.3\%) and $35(46.7 \%)$ of the 75 isolates, respectively; for posaconazole all 35 isolates were $\mathrm{C}$ glabrata. Taking into account all 464 isolates, a correlation $\left(r^{2}>0.87\right)$ was observed between increasing fluconazole MICs and the geometric mean MICs for itraconazole, voriconazole, posaconazole and ravuconazole (Figure 1). None of the isolates had a caspofungin MIC exceeding $1 \mathrm{mg} / \mathrm{L}$. Discrepancies in interpretation, when comparing the $24 \mathrm{~h}$ and the $48 \mathrm{~h}$ MICs for flucytosine, itraconazole, fluconazole and voriconazole, are shown in Table 3.

\section{DISCUSSION}

The average annual incidence of candidemia in Quebec during our two-year study period was three per 100,000 population. This rate is comparable with a previous 1992 to 1994 Canadian study (17) that reported incidence rates varying from 1.2 to 5.1 in metropolitan Hamilton, Burlington and Ottawa in the province of Ontario, and in the province of Manitoba. In a more recent population-based study between 1999 and 2004 in Alberta, Laupland et al (15) reported an incidence rate of 2.8 per 100,000 population for central nervous system and bloodstream infections. Overall, rates reported in Canadian studies are lower than those from the United States which are, on average, two to three times higher

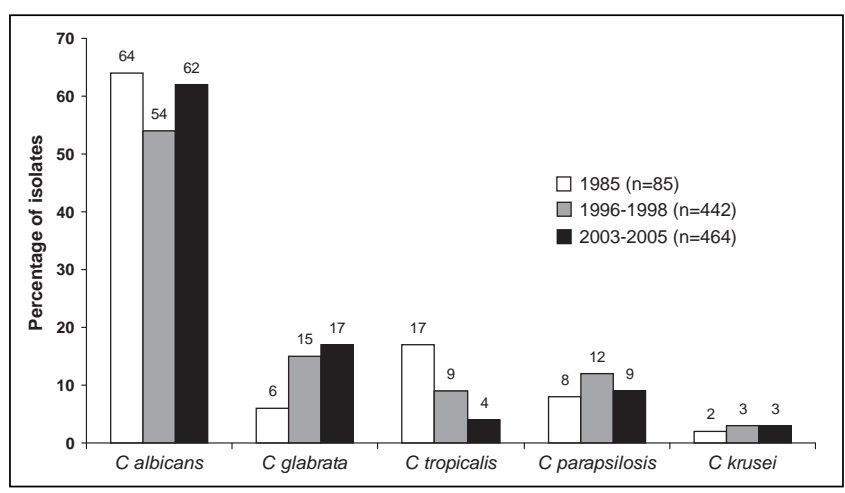

Figure 1) Variation in the distribution of the five most frequently isolated species of Candida according to three Quebec surveillance studies in 1985, 1996 to 1998, and 2003 to 2005

$(7,8,11)$. The reasons for these discrepancies remain unclear, but could be related to variations in patient population, patient management or differences in study methodologies (7).

In North America and in most countries worldwide, $\mathrm{C}$ albicans continues to be the single most common species causing candidemia (7,27-29). Compared with the findings of our 1996 to 1998 , and 1985 studies, the present results indicate little variation in the frequency of $\mathrm{C}$ albicans isolates and a slight, but gradual, increase in number of $\mathrm{C}$ glabrata isolates with a commensurate decrease in C tropicalis (Figure 1) $(18,19)$. With the decline in frequency of $\mathrm{C}$ tropicalis, $\mathrm{C}$ parapsilosis has become the third most prevalent invasive Candida species observed in Quebec. These variations are congruent with the 
TABLE 3

Discrepancies in interpretation when comparing $24 \mathrm{~h}$ to $48 \mathrm{~h}$ minimum inhibitory concentrations for four antifungal agents

\begin{tabular}{|c|c|c|c|c|c|c|c|c|}
\hline \multirow{2}{*}{$\begin{array}{l}\text { Candida species } \\
\text { (number of isolates) }\end{array}$} & \multicolumn{2}{|c|}{ Flucytosine } & \multicolumn{2}{|c|}{ Itraconazole } & \multicolumn{2}{|c|}{ Fluconazole } & \multicolumn{2}{|c|}{ Voriconazole } \\
\hline & Minor $^{*}$ & Very major ${ }^{\dagger}$ & Minor & Very major & Minor & Very major & Minor & Very major \\
\hline C albicans $(n=288)$ & 2 & 1 & 1 & - & 1 & - & - & - \\
\hline C glabrata $(n=78)$ & - & - & 5 & 1 & 50 & - & - & - \\
\hline$C$ parapsilosis $(\mathrm{n}=43)$ & 1 & - & 10 & - & - & - & - & - \\
\hline C tropicalis $(\mathrm{n}=21)$ & - & - & 4 & - & 1 & $2^{\ddagger}$ & - & $2^{\ddagger}$ \\
\hline C lusitaniae $(n=13)$ & - & - & 2 & - & - & - & - & - \\
\hline C krusei $(\mathrm{n}=12)$ & 1 & - & 3 & - & 6 & - & - & - \\
\hline C dubliniensis $(\mathrm{n}=3)$ & - & - & - & - & - & - & - & - \\
\hline C guilliermondii $(\mathrm{n}=3)$ & - & - & 1 & - & 1 & - & - & - \\
\hline C pelliculosa $(\mathrm{n}=2)$ & - & - & - & - & - & - & - & - \\
\hline$C$ kefyr $(\mathrm{n}=1)$ & - & - & - & - & - & - & - & - \\
\hline Total $(n=464)$ & 4 & 1 & 26 & 1 & 59 & 2 & - & 2 \\
\hline
\end{tabular}

"Minor error - intermediate or susceptible dose-dependant isolate interpreted as susceptible or, resistant isolate interpreted as intermediate or susceptible dosedependant; † Very major error - resistant isolate interpreted as susceptible; ${ }^{\ddagger}$ Low-high isolates - errors attributed to $48 \mathrm{~h}$ and not $24 \mathrm{~h}$ readings

observations made in some longitudinal studies $(9,29,30,31)$. C dubliniensis is a newly reported species in candidemia surveillance studies. The rate of isolation of $0.6 \%$ observed during our study period is similar to those of $0.8 \%$ and $0.2 \%$ reported by Hajjeh et al (7) and Pfaller et al (31), respectively. It has been suggested that the higher prevalence of $\mathrm{C}$ glabrata reported in older patients may be institution dependant (7). In our study, C glabrata accounted for $21.6 \%$ of isolates recovered in elderly patients (65 years of age or older) compared with $3.2 \%$ in infants (one year of age or younger); the difference was statistically significant. The 47 bloodstream isolates recovered in older patients originated from 22 different hospitals with diversified patient populations, suggesting that the high prevalence of C glabrata in the elderly is likely independent of hospital settings. Our observations on the high prevalence of $\mathrm{C}$ parapsilosis in the neonatal and infant population concur with those of previous studies $(7,8,29)$.

At the present time, concerns over the inability of the CLSI method M27-A to adequately detect amphotericin B resistance remain, and the clinical significance of in vitro resistance to amphotericin $\mathrm{B}$ has yet to be established $(21,22,25,32)$. As reported in other studies, our MIC data indicate little in vitro resistance to this drug $(7,33)$. Decreased susceptibility to amphotericin B was mainly seen in C glabrata, C krusei and C lusitaniae with $90 \%$ of the isolates tested inhibited at a MIC of $1 \mathrm{mg} / \mathrm{L}$. These species were previously observed to be innately less susceptible in vitro to amphotericin B, and Goldman et al (34) have reported that a significantly better response to $\mathrm{C}$ krusei infections was obtained when patients were treated with amphotericin B dosages greater than $1 \mathrm{mg} / \mathrm{kg} / \mathrm{day}$ compared with patients receiving lower dosages $(8,35,36)$. The proportion of $\mathrm{C}$ albicans isolates resistant to flucytosine was $2.4 \%$ and is essentially the same as that previously observed in our 1996 to 1998 survey (3\%) (18). Similar results have been reported in other studies $(7,28)$.

Of the 272 patients evaluable for antifungal treatment after the onset of their candidemia, $80 \%$ were treated with fluconazole. The species distribution and susceptibility profiles of the isolates indicate that the widespread use of this antifungal agent has not resulted in the selection of Candida species known to be less susceptible to fluconazole, such as C krusei and C glabrata (Table 2), nor in the increase of azole resistance in other species. This observation is essentially similar to our 1996 to 1998 survey, and agrees with many other reports (18,27-30). Three $\mathrm{C}$ tropicalis isolates were found to have $48 \mathrm{~h}$ MICs exceeding $64 \mathrm{mg} / \mathrm{L}$. One of these exhibited a fluconazole MIC of $32 \mathrm{mg} / \mathrm{L}$ at $24 \mathrm{~h}$ and was from a solid organ transplant patient exposed to fluconazole before the diagnosis of candidemia. However, the other two isolates, which were from patients who were not exposed previously to azoles, exhibited low MICs of $0.5 \mathrm{mg} / \mathrm{L}$ and $1.0 \mathrm{mg} / \mathrm{L}$ at $24 \mathrm{~h}$. These two isolates exhibiting 'low-high' MICs at $24 \mathrm{~h}$ and $48 \mathrm{~h}$ of incubation were reported as susceptible in accordance with recommendations from two in vivo investigations of this phenomenon $(37,38)$. Interestingly, for one of these isolates, the same phenomenon was observed with all four other azoles tested in our survey. Although only four (5.1\%) isolates of C glabrata were resistant to fluconazole, 59 (75.6\%) had reduced susceptibility to this antifungal. Similar results have been reported and support the hypothesis that fluconazole should not be the initial treatment of choice for C glabrata candidemia (7). It has been suggested that previous exposure to fluconazole may select for C krusei or C glabrata candidemia (27). Our results are supportive of this observation. Six C krusei strains and six C glabrata strains (24.0\% each) were isolated from 12 of 25 patients previously exposed to fluconazole, compared with 50 isolates of C glabrata (16.9\%) and six of C krusei (2.0\%) isolated from 56 of 296 nonexposed patients.

With regard to itraconazole, none of the 75 Candida isolates with reduced susceptibility to fluconazole (MIC $16 \mathrm{mg} / \mathrm{L}$ or greater) were susceptible to this drug (MIC $0.12 \mathrm{mg} / \mathrm{L}$ or lower). However, Pfaller et al (39) recently suggested that MICs of $1 \mathrm{mg} / \mathrm{L}$ or lower may better reflect 'susceptibility' in invasive candidiasis, due to the higher serum concentrations achievable with the new nanocrystal intravenous formulation of itraconazole. Given this new threshold, 58.0\% of our isolates with reduced susceptibility to fluconazole would be considered susceptible to itraconazole. Furthermore, our observations are similar to those of Pfaller et al, with all four of our C glabrata isolates resistant to fluconazole also resistant to itraconazole, and only three of 12 (25\%) isolates of C krusei showing complete cross-resistance. Overall, $92.7 \%$ of all isolates in our study were inhibited by $1 \mathrm{mg} / \mathrm{L}$ of itraconazole or lower.

Our study includes susceptibility testing with new antifungal agents. To date, there have been few population-based studies $(15,32)$ reporting on the activity of these agents against Candida bloodstream isolates. As observed in these previous 
studies, voriconazole, posaconazole and ravuconazole all displayed a broad spectrum of activity against Candida species recovered in our survey. Of the 75 isolates with reduced susceptibility to fluconazole (MIC $16 \mathrm{mg} / \mathrm{L}$ or greater), 93\% were susceptible to voriconazole. However, as observed also by Pfaller et al (40), decreasing susceptibility to fluconazole was most often associated with decreasing susceptibility to voriconazole and only five of 12 (42\%) fluconazole-resistant isolates were susceptible to voriconazole. All C krusei isolates were inhibited by $1 \mathrm{mg} / \mathrm{L}$ of voriconazole or lower, ravuconazole and posaconazole. Also, 94.9\%, $93.6 \%$ and $53.3 \%$ of isolates of C glabrata were inhibited by $1 \mathrm{mg} / \mathrm{L}$ or lower of each of these drugs respectively. Among the 11 isolates with fluconazole MICs $64 \mathrm{mg} / \mathrm{L}$ or greater, two were resistant to voriconazole (MIC $4 \mathrm{mg} / \mathrm{L}$ or greater) and three exhibited MICs $2 \mathrm{mg} / \mathrm{L}$ or greater for both ravuconazole and posaconazole. Strong correlations $(\mathrm{P}>0.95)$ were observed when the geometric mean MICs of all 464 isolates for voriconazole, itraconazole posaconazole and ravuconazole are categorized according to fluconazole MICs (Figure 2). This does not necessarily indicate a trend toward full crossresistance, but shows that the mechanisms responsible for increasing fluconazole MICs almost invariably have some impact on other azoles. Caspofungin was active in vitro against all Candida species tested in this study. All MICs, including those of isolates resistant to fluconazole, were $1 \mathrm{mg} / \mathrm{L}$ or lower. These results concur with those of the population-based studies of Laupland et al (15) and CuencaEstrella et al (33), as well as those of a global surveillance study $(24,32,41)$. Our MIC data also shows a shift toward higher values for $\mathrm{C}$ parapsilosis relative to $\mathrm{C}$ albicans.

Our survey also provides information on the discrepancies in MICs noted with triazoles when comparing $24 \mathrm{~h}$ and $48 \mathrm{~h}$ readings. Reading discrepancies between $24 \mathrm{~h}$ and $48 \mathrm{~h}$ had little impact on the final categorical interpretation for $\mathrm{C}$ albicans and $\mathrm{C}$ tropicalis, but resulted in minor errors, mainly with C glabrata, C parapsilosis and C krusei. Overall, 59 minor errors were observed with fluconazole, but this number would be reduced to 11 with $24 \mathrm{~h}$ breakpoints set at one dilution lower than the actual $48 \mathrm{~h}$ breakpoints. These observations concur with those of Espinel-Ingroff et al (42) who have recently suggested that the reporting of $24 \mathrm{~h}$ MICs for azoles should be considered in the next revised version of the CLSIs M27 reference method. The only very major errors observed were with the two 'low-high' $\mathrm{C}$ tropicalis isolates resistant to fluconazole at $48 \mathrm{~h}$ and susceptible at $24 \mathrm{~h}$. Murine models of invasive candidiasis have shown that isolates with such behaviour should be considered susceptible rather than resistant $(37,38)$.

\section{CONCLUSION}

The present surveillance program provides a population-based description of candidemia in Quebec, and shows little variation in species distribution and antifungal resistance profiles compared with results from our 1996 to 1998 study. Resistance to fluconazole in $\mathrm{C}$ albicans remains rare and the frequency of

\section{REFERENCES}

1. Beck-Sagué C, Jarvis WR. Secular trends in the epidemiology of nosocomial fungal infections in the United States, 1980-1990. National Nosocomial Infections Surveillance System. J Infect Dis 1993; 167:1247-51.

2. Edmond MB, Wallace SE, McClish DK, Pfaller MA, Jones RN, Wenzel RP. Nosocomial bloodstream infections in United States hospitals: A three-year analysis. Clin Infect Dis 1999;29:239-44.

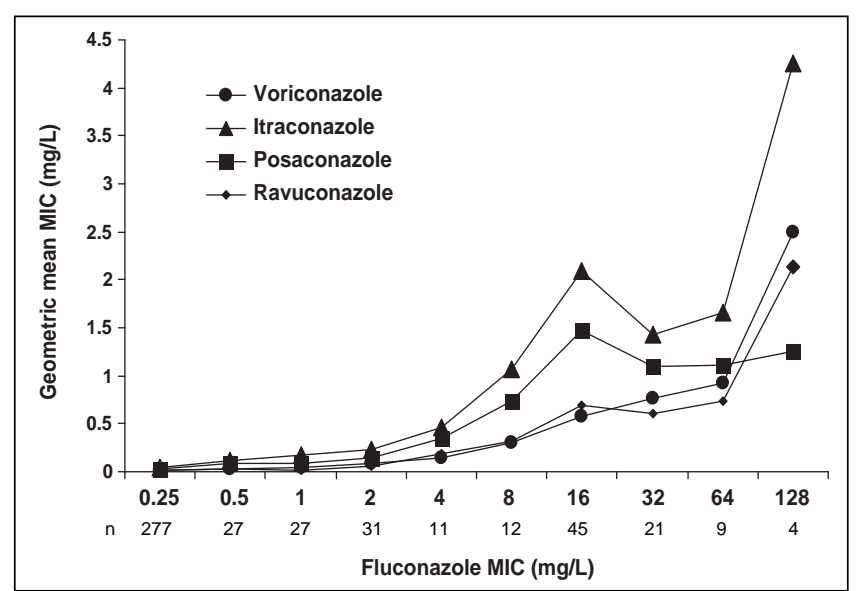

Figure 2) Geometric mean minimum inhibitory concentrations (MICs) of 464 Candida species isolates for four azole antifungal agents categorized according to fluconazole MICs

isolation of intrinsically azole-resistant $\mathrm{C}$ krusei remains low. At the present time, resistance of $\mathrm{C}$ albicans to fluconazole appears to be essentially transitory and associated with long-term exposure to this drug. In Quebec, the systematic susceptibility testing of Candida bloodstream isolates appears to be warranted only in patients previously exposed or undergoing long-term azole treatment. Also, through prompt identification, over $20 \%$ of our candidemic isolates would have had been identified as species with known intrinsic resistance or lesser susceptibility to fluconazole.

ACKNOWLEDGEMENTS: The authors thank those who provided isolates and clinical data for the present study. Participants contributing to the study included: Isabelle Alarie, James Allen, Pierre Auger, Marcel Behr, Brigitte Bélanger, Guy Boivin, Jean Bouchard, Doris Bourbeau, Gilbert Cérat, Guy Chamberland, Linda Cohen, Louise Côté, Sonya d'Agnese, André Dascal, Anne-Marie Demers, Louise Dion, Mélanie Dionne, Joe Dylewsky, Daniel Eymard, Guylaine Filion, Lise-Andrée Galarneau, Sylviane Geoffroy, Lucie Gilbert, Louise Gingras, Marie Gourdeau, Monique Goyette, Chris Greenaway, Magued Ishak, André Jolicoeur, Marie Jolivet, Kathleen Knowles, Lajos Kovacs, Céline Laferrière, Pierre Lebel, Lucie Mailloux-Cérat, Mirabelle Kelly, Jane McDonald, Alain Martel, Jack Mendelson, Sophie Michaud, Mark Miller, Serge Montplaisir, Isabelle Morissette, Gilles Murray, Tuyen Nguyen, Grégoire Noël, Alain Paradis, Jean-François Paradis, Gilles Pelletier, Lorraine Pelletier, Jacques Péloquin, Alain Piché, Cécile Plouffe, Louise Poirier, David Portnoy, Joseph Portnoy, Caroline Quach, Alain Renaud, Sylvie Ricard, Annie Rivest, Michel Robillard, Daniel Robitaille, Josée Robitaille, Céline Rousseau, Mélanie Masse, Earl Rubin, Marie St-Yves, Miguelle Sanchez, Christian Sinave, Leon Solomon, Christine Tanguay, Sylvie Théoret, Silvana Trifiro, Anik Tremblay, Louise Tremblay, Suzanne Trottier, Nathalie Turgeon, Pierre Turgeon, Brian Ward, Ariane Yechouron and R Zavalkoff. The authors also thank Christiane Dion and Danielle Beauchesne for their excellent technical assistance, and Réjean Dion for providing support with statistical analyses.

3. Golan Y, Wolf MP, Pauker SG, Wong JB, Hadley S. Empirical antiCandida therapy among selected patients in the intensive care unit: A cost-effectiveness analysis. Ann Intern Med 2005;143:857-69.

4. Morgan J, Meltzer MI, Plikaytis BD, et al. Excess mortality, hospital stay, and cost due to candidemia: A case-control study using data from population-based candidemia surveillance. Infect Control Hosp Epidemiol 2005;26:540-7. (Erratum in 2005;26:675). 
5. Zaoutis TE, Argon J, Chu J, Berlin JA, Walsh TJ, Feudtner C. The epidemiology and attributable outcomes of candidemia in adults and children hospitalized in the United States: A propensity analysis. Clin Infect Dis 2005;41:1232-9.

6. Almirante B, Rodríguez D, Park BJ, et al; Barcelona Candidemia Project Study Group. Epidemiology and predictors of mortality in cases of Candida bloodstream infection: Results from populationbased surveillance, Barcelona, Spain, from 2002 to 2003.

J Clin Microbiol 2005;43:1829-35.

7. Hajjeh RA, Sofair, AN, Harrison LH, et al. Incidence of bloodstream infections due to Candida species and in vitro susceptibilities of isolates collected from 1998 to 2000 in a population-based active surveillance program. J Clin Microbiol 2004;42:1519-27.

8. Kao AS, Brandt ME, Pruitt WR, et al. The epidemiology of candidemia in two United States cities: Results of a populationbased active surveillance. Clin Infect Dis 1999;29:1164-70.

9. Sandven P. Epidemiology on candidemia. Rev Iberoam Micol 2000;17:73-81.

10. Asmundsdóttir LR, Erlendsdóttir H, Gottfredsson M. Increasing incidence of candidemia: Results from a 20-year nationwide study in Iceland. J Clin Microbiol 2002;40:3489-92.

11. Diekema DJ, Messer SA, Brueggemann AB, et al. Epidemiology of candidemia: 3 -year results from the emerging infections and the epidemiology of Iowa organisms study. J Clin Microbiol 2002;40:1298-302.

12. Poikonen E, Lyytikäinen $O$, Anttila VJ, Ruutu P. Candidemia in Finland, 1995-1999. Emerg Infect Dis 2003;9:985-90.

13. Sandven P, Bevanger L, Digranes A, Gaustad P, Haukland HH, Steinbakk M. Constant low rate of fungemia in Norway, 1991 to 1996. The Norwegian Yeast Study Group. J Clin Microbiol 1998;36:3455-9.

14. Karlowsky JA, Zhanel GG, Klym KA, Hoban DJ, Kabani AM. Candidemia in a Canadian tertiary care hospital from 1976 to 1996. Diagn Microbiol Infect Dis 1997;29:5-9.

15. Laupland KB, Gregson DB, Church DL, Ross T, Elsayed S. Invasive Candida species infections: A 5 year population-based assessment. J Antimicrob Chemother 2005;56:532-7.

16. Macphail GL, Taylor GD, Buchanan-Chell M, Ross C, Wilson S, Kureishi A. Epidemiology, treatment and outcome of candidemia: A five-year review at three Canadian hospitals. Mycoses 2002:45:141-5.

17. Yamamura DL, Rotstein C, Nicolle LE, Ioannou S. Candidemia at selected Canadian sites: Results from the Fungal Disease Registry, 1992-1994. Fungal Disease Registry of the Canadian Infectious Disease Society. CMAJ 1999;23:493-9.

18. St-Germain G, Laverdière $M$, Pelletier R, et al. Prevalence and antifungal susceptibility of 442 Candida isolates from blood and other normally sterile sites: Results of a 2-year (1996 to 1998) multicenter surveillance study in Quebec, Canada. J Clin Microbiol 2001;39:949-53.

19. St-Germain G. [Étude de 100 souches de levures isolées de spécimens cliniques normalement stériles.] Bull Soc Fr Mycol Med 1988;17:309-12

20. Al Mosaid A, Sullivan D, Salkin IF, Shanley D, Coleman DC. Differentiation of Candida dubliniensis from Candida albicans on Staib agar and caffeic acid-ferric citrate agar. J Clin Microbiol 2001;39:323-7.

21. Pfaller MA. Reference method for broth dilution antifungal susceptibility testing of yeasts. National Committee for Clinical Laboratory Standards. Approved standard M27-A2, 2nd edn, 2005.

22. Rex JH, Pfaller, MA, Walsh TJ, et al. Antifungal susceptibility testing: Practical aspects and current challenges. Clin Microbiol Rev 2001;14:643-58.

23. Odds FC, Motyl M, Andrade R, et al. Interlaboratory comparison of results of susceptibility testing with caspofungin against Candida and Aspergillus species. J Clin Microbiol 2004:42:3475-82.

24. Pfaller MA, Messer SA, Boyken L, et al. Further standardization of broth microdilution methodology for in vitro susceptibility testing of caspofungin against Candida species by use of an international collection of more than 3,000 clinical isolates. J Clin Microbiol 2004:42:3117-9.

25. Rex JH, Cooper CR Jr, Merz WG, Galgiani JN, Anaissie EJ. Detection of amphotericin B-resistant Candida isolates in a broth-based system. Antimicrob Agents Chemother 1995;39:906-9.
26. Clinical and Laboratory Standards Institute. Quality control minimal inhibitory concentration (MIC) limits for broth microdilution and MIC interpretive breakpoints. Informational supplement M27-S2, 2nd edn, 2005.

27. Colombo AL, Nucci M, Park BJ, et al; Brazilian Network Candidemia Study. Epidemiology of candidemia in Brazil: A nationwide sentinel surveillance of candidemia in eleven medical centers. J Clin Microbiol 2006;44:2816-23.

28. Messer SA, Jones RN, Fritsche TR. International surveillance of Candida spp and Aspergillus spp: Report from the SENTRY antimicrobial surveillance program (2003). J Clin Microbiol 2006;44:1782-7.

29. Sandven P, Bevanger L, Digranes A, et al. Candidemia in Norway (1991 to 2003): Results from a nationwide study. J Clin Microbiol 2006;44:1977-81.

30. Pfaller MA, Diekema DJ; International Fungal Surveillance Participant Group. Twelve years of fluconazole in clinical practice: Global trends in species distribution and fluconazole susceptibility of bloodstream isolates of Candida. Clin Microbiol Infect 2004;10(Suppl 1):11-23

31. Pfaller MA, Diekema DJ, Gibbs, et al; Global Antifungal Surveillance Study. Results from the ARTEMIS DISK Global Antifungal Surveillance study, 1997 to 2005: An 8.5-year analysis of susceptibilities of Candida species and other yeast species to fluconazole and voriconazole determined by CLSI standardized disk diffusion testing. J Clin Microbiol 2007;45:1735-45.

32. Park BJ, Arthington-Skaggs BA, Hajjeh RA, et al. Evaluation of amphotericin B interpretive breakpoints for Candida bloodstream isolates by correlation with therapeutic outcome. Antimicrob Agents Chemother 2006;50:1287-92.

33. Cuenca-Estrella M, Rodriguez D, Almirante B, et al; the Barcelona Candidemia Project Study Group. In vitro susceptibilities of bloodstream isolates of Candida species to six antifungal agents: Results from a population-based active surveillance programme, Barcelona, Spain, 2002-2003. J Antimicrob Chemother 2005;55:194-9.

34. Goldman M, Pottage JC Jr, Weaver DC. Candida krusei fungemia. Report of four cases and review of the literature. Medicine (Baltimore) 1993;72:143-50.

35. Fidel PL Jr, Vazquez JA, Sobel JD. Candida glabrata: Review of epidemiology, pathogenesis, and clinical disease with comparison to C albicans. Clin Microbiol Rev 1999;12:80-96.

36. Krogh-Madsen M, Arendrup MC, Heslet L, Knudsen JD. Amphotericin B and caspofungin resistance in Candida glabrata isolates recovered from a critically ill patient. Clin Infect Dis 2006;42:938-44.

37. Arthington-Skaggs BA, Warnock DW, Morrison CJ. Quantitation of Candida albicans ergosterol content improves the correlation between in vitro antifungal susceptibility test results and in vivo outcome after fluconazole treatment in a murine model of invasive candidiasis. Antimicrob Agents Chemother 2000;44:2081-5.

38. Rex JH, Nelson PW, Paetznick VL, Lozano-Chiu M, Espinel-Ingroff A, Anaissie EJ. Optimizing the correlation between results of testing in vitro and therapeutic outcome in vivo for fluconazole by testing critical isolates in a murine model of invasive candidiasis. Antimicrob Agents Chemother 1998;42:129-34.

39. Pfaller MA, Boyken L, Hollis RJ, Messer SA, Tendolkar S, Diekema DJ. In vitro susceptibilities of clinical isolates of Candida species, Cryptococcus neoformans, and Aspergillus species to itraconazole: Global survey of 9359 isolates tested by Clinical and Laboratory Standards Institute broth microdilution methods. J Clin Microbiol 2005;43:3807-10.

40. Pfaller MA, Messer SA, Boyken L, et al. Use of fluconazole as a surrogate marker to predict susceptibility and resistance to voriconazole among 13,338 clinical isolates of Candida spp. Tested by clinical and laboratory standards institute-recommended broth microdilution methods. J Clin Microbiol 2007;45:70-5.

41. Pfaller MA, Boyken L, Hollis RJ, Messer SA, Tendolkar S, Diekema DJ. In vitro susceptibilities of Candida spp. to caspofungin: Four years of global surveillance. J Clin Microbiol 2006;44:760-3.

42. Espinel-Ingroff A, Barchiesi F, Cuenca-Estrella M, et al. Comparison of visual 24-hour and spectrophotometric 48-hour MICs to CLSI reference microdilution MICs of fluconazole, itraconazole, posaconazole, and voriconazole for Candida spp: A collaborative study. J Clin Microbiol 2005;43:4535-40. 


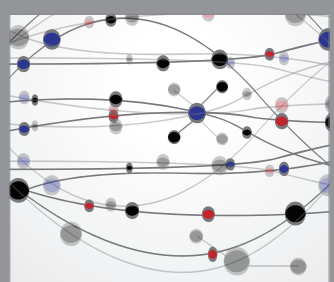

The Scientific World Journal
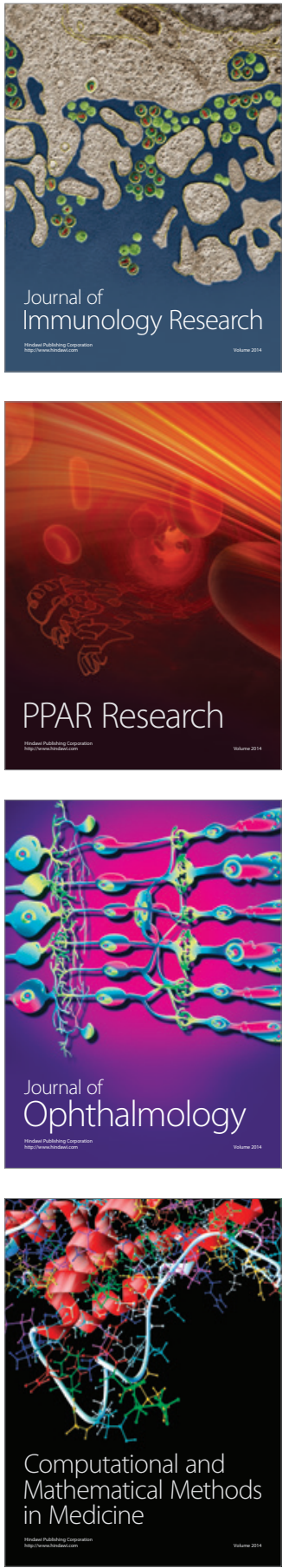

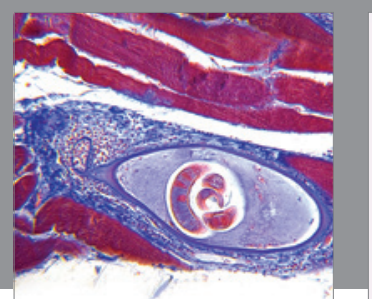

Gastroenterology Research and Practice

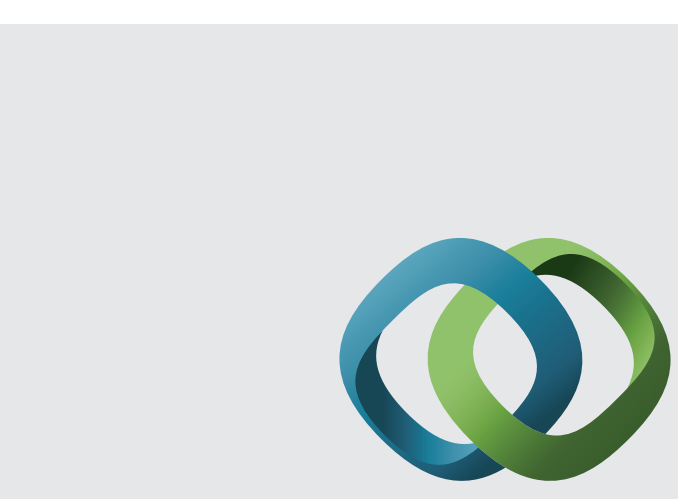

\section{Hindawi}

Submit your manuscripts at

http://www.hindawi.com
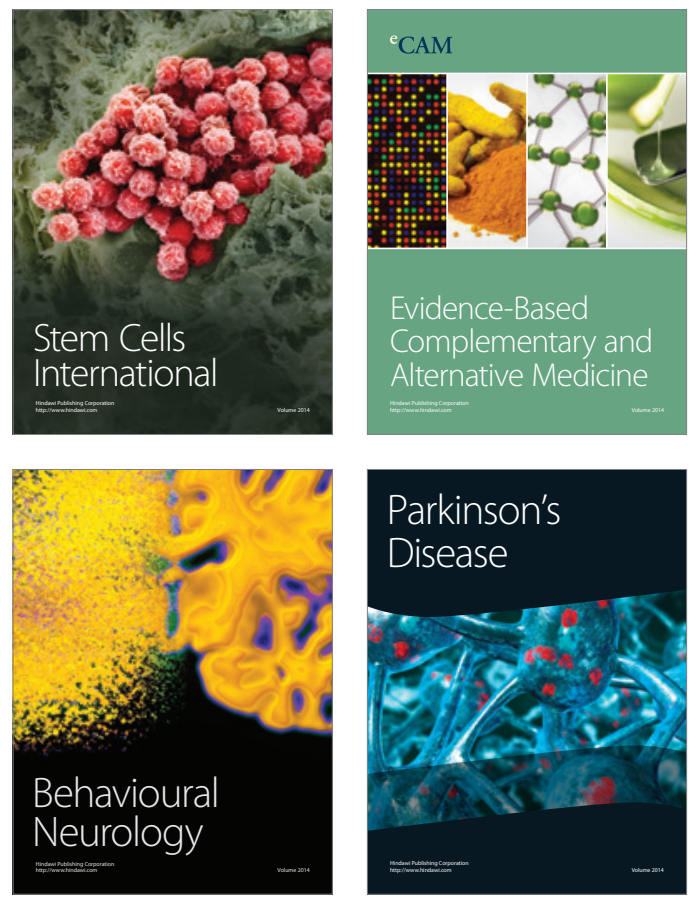
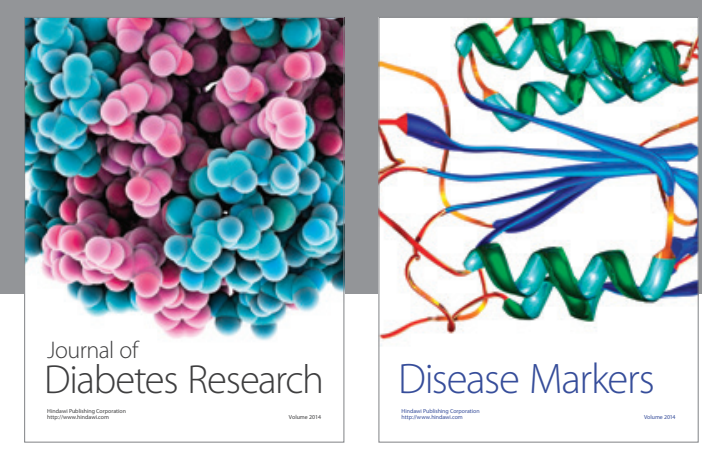

Disease Markers
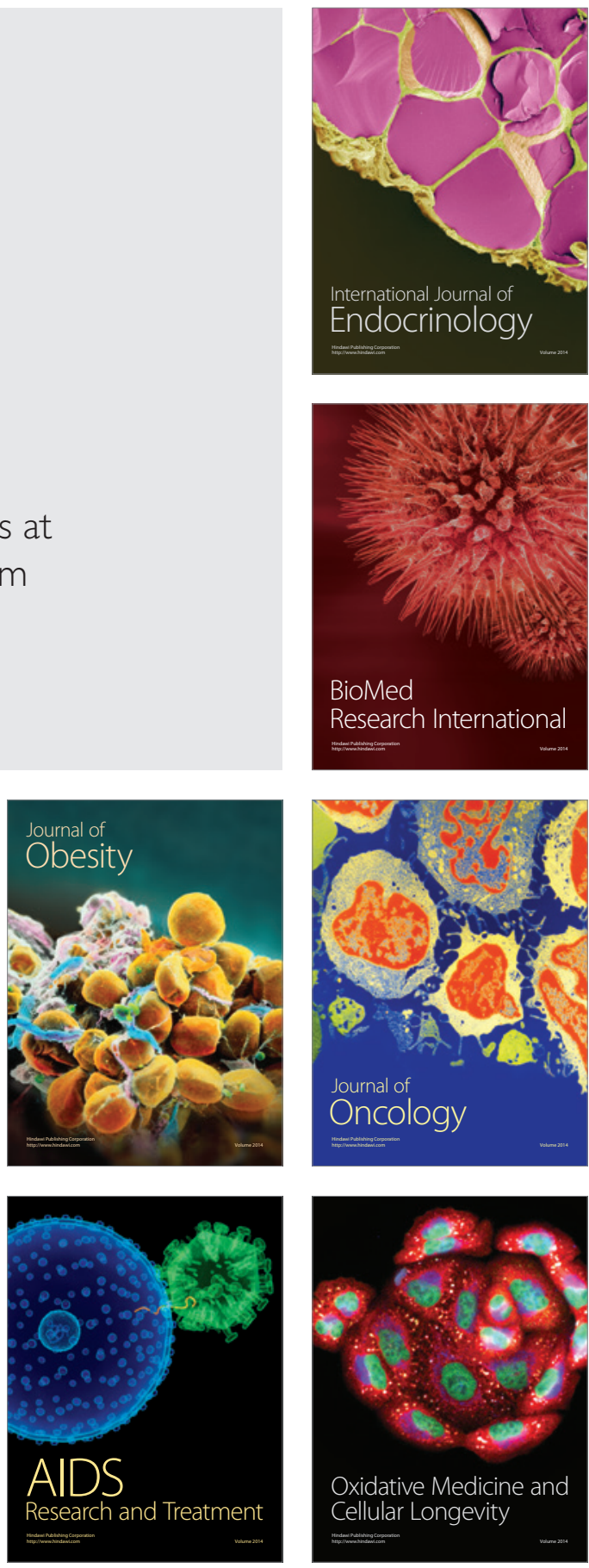